\title{
RECHERCHES SÉDIMENTOLOGIQUES ET GÉOLOGIQUES EN MANCHE CENTRALE
}

\author{
par Claude LaRsonneur
}

Dans le cadre de nos recherches en Manche centrale et orientale le navire océanographique "Thalassa » (') nous a apporté son concours pour prospecter par dragages une zone rectangulaire située au large de Cherbourg et comprise entre $0^{\circ} 30^{\circ}$ et $2^{\circ}$ de longitude ouest et $50^{\circ} 05^{\prime}$ et $50^{\circ} 14$ de latitude nord (fig. 1).

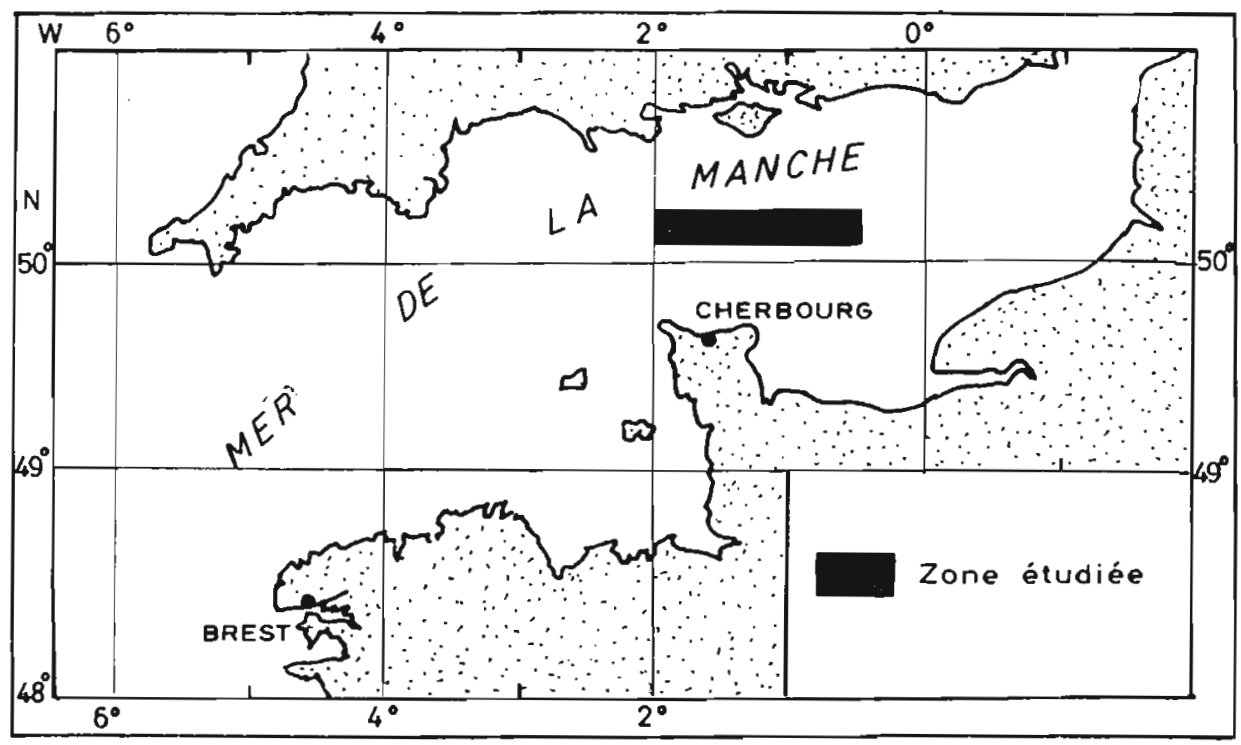

FIG. 1. - Situation géographique de la zone éfudiée.

Nos prélèvements de fonds, réalisés avec une drague Rallier du Baty de $47 \mathrm{~cm}$ de diamètre, ont été effectués tous les 3 milles. A chaque station la position du bateau était définie en coordonnées Decca et la profondeur lue sur la bande d'un écho-sondeur. La position de nos stations a été reportée sur la carte de la figure 2 ainsi que la topographie des fonds extraite de la carte 5085 du Service Hydrographique de la Marine.

\section{Etude des sédiments}

Dans cette zone où la vitesse des courants de marée peut atteindre 4 nceuds en marée de vive eau, les fonds sédimentaires sont assez peu variés. La drague ramène toujours une très forte proportion d'éléments supérieurs à $2 \mathrm{~cm}$ et, comme l'ont montré de nombreuses études antérieures : L. Dangeard (12), L. Berthois et J. Furnestin (2), J. Bourcart (8), L. et C. Berthois (1) et $G$. Boillot (4 et 6 ), c'est là un phénomène assez général dans la Manche.

(1) Pour ce trés précieux concours, nous adressons notre reconnaissance à M. J. Furnestrn. Directeur de l'Institut scientifique et technique des Péches maritimes, ainsi qu'au commandant R. Brenot et à l'équipage du N.O. "Thalassa » 


\section{1. - Description des sédiments.}

Une passoire munie de trous de $2 \mathrm{~cm}$ de diamètre nous a permis d'effectuer une première séparation du sédiment sur le bateau. Pour la partie inférieure à $20 \mathrm{~mm}$, composée de sables et de graviers d'après les limites dimensionnelles de A. Callleux (10), nous avons fait une prise homogène en notant la fraction volumétrique recueillie: sa pesée permettant par la suite de connaitre le poids sec dragué.

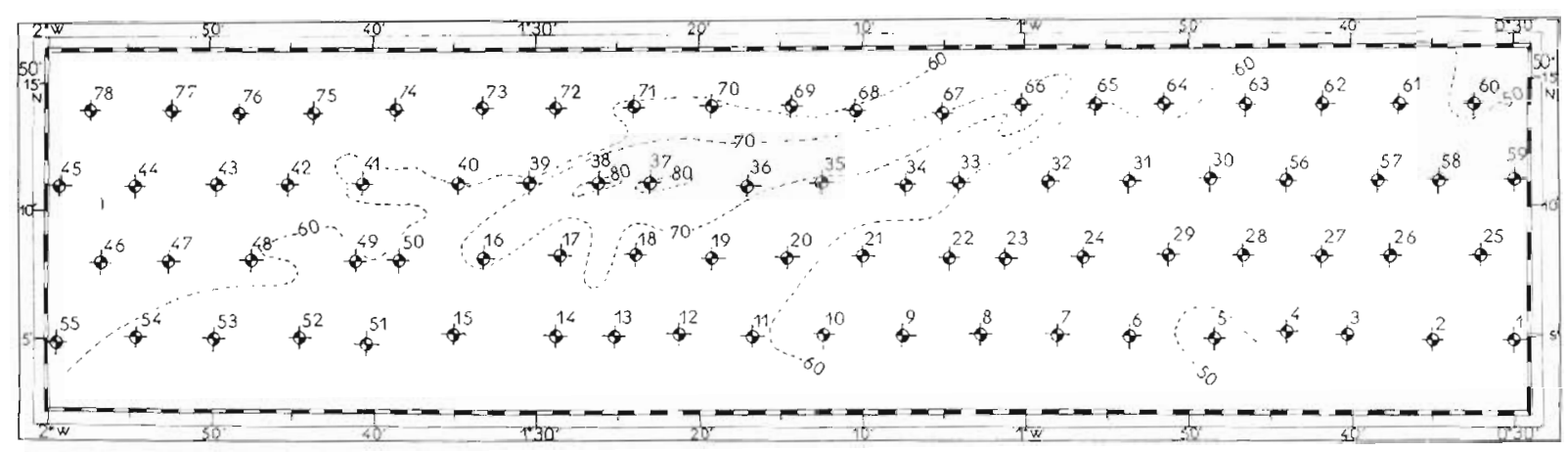

FIG. 2. - Position des stations du N.O. «Thalassa » et fond topographique d'apress la carte 5085 du Service hydrographique de la Marine.

La partie supérieure à $20 \mathrm{~mm}$, a été recueillie entière lorsqu'elle était peu importante, ou fractionnée sur place comme il sera indiqué plus loin. Après pesée des différentes fractions, de nombreux silex ont été rejetés et les autres roches conservées.

Les éléments supérieurs à $20 \mathrm{~cm}$ (blocs) étant extrêmement rares dans nos dragages, le refus de notre passoire à trous de $2 \mathrm{~cm}$ correspond à la tranche galets ou cailloux de A. CaIlleux (10). Pour J. Bourcart (7), le terme cailloux a un sens plus large puisquil désigne la phase supérieure à $2 \mathrm{~mm}$. Nous préférons donc utiliser le terme galet (1) pour nommer la fraction granulométrique comprise entre 2 et $20 \mathrm{~cm}$.

Dans la plupart de nos dragages, la proportion de galets est supérieure à 50 \% et, d'après les définitions de G. Boillor (3), ces fonds peuvent être rattachés à la zone des cailloutis résiduels (zone 1). Seules les stations 25 et 61 qui ont fourni respectivement 42,5 et $43,5 \%$ de galets et où le sédiment renferme plus de $25 \%$ de gravier siliceux sans les cailloux (respectivement 63 et $74 \%$ ) sont à placer dans la zone II : graviers résiduels. Toutefois, il existe une assez grande variété de sédiments dans la zone des cailloutis et nous avons voulu en rendre compte par notre cartographie des fonds. Dans ce but, nous avons subdivisé la zone I en 4 sous-zones définies comme suit:

sous-zone $I_{1} \ldots \ldots$ de 95 à $100 \%$ de galets : cailloutis purs

sous-zone $I_{2} \ldots \ldots$ de 90 à $95 \%$ de galets : cailloutis légèrement graveleux,

sous $-z$ one $I_{3} \ldots \ldots$ de 75 à $90 \%$ de galets : cailloutis moyennement graveleux,

sous-zone $I_{4} \ldots \ldots$ de 50 à $75 \%$ de galets : cailloutis graveleux.

De plus, les stations ont été affectées de la lettre «s $s$ lorsque le sédiment sans les galets contenait plus de $25 \%$ de sable, «ss 》 lorsque cette teneur était supérieure à $50 \%$ et «sss»lorsqu'elle dépassait $75 \%$. On peut donc successivement remplacer l'adjectif graveleux de la définition par les termes: gravelo-sableux, sablo-graveleux et sableux. Les sous-zones $I_{1}, I_{2}$ et $I_{;}$correspondent sensiblement à la sous-zone Ia de G. Boillot et la sous-zone I, à la sous-zone Ib du même auteur.

(1) Souvent le terme galet implique un certain façonnement mais on ne voit pas à partir de quel degré d’usure on peut commencer à l'utiliser. D'ailleurs, la morphologie dépend de la taille et, comme l'écrivait J. Boukcart en 1941 (7) : «Le seul terrain solide pour y asseoir une classification est celui de la dimension des particules. galet n'a donc pour nous qu'une signification de taille. On peut ensuite caractériser la forme de ces élements en utilisant les indices définis par A. Carlleux. 
Dans cette région, le sédiment se caractérise encore par la taille des galets et nous avons voulu tenir compte de ce caractère dans la représentation des fonds. Pour chaque dragage nous avons effectué une granulométrie des galets selon la méthode préconisée par P. HommeriL (16). Ceux-ci sont répartis d'après leur longueur en 7 classes dont les limites varient en progression géométrique de raison $\sqrt{ } 2$; ces classes sont les suivantes :

\begin{tabular}{|c|c|c|c|c|}
\hline asse & $1: \mathrm{d}$ & 21 à & 30 & \\
\hline$»$ & $2: d$ & & 42 & \\
\hline$\gg$ & $3: 0$ & 42 à & 60 & \\
\hline$»$ & $4: 0$ & 60 à & 84 & \\
\hline
\end{tabular}

classe $5:$ de 84 à $120 \mathrm{~mm}$
$\gg \quad 6:$ de 120 à $168 \%$
$\gg \quad 7:$ de 168 à $240 \gg$

Chaque lot est ensuite pesé, ce qui permet de construire la courbe cumulative et de définir la médiane. Celle-ci varie de $4 \mathrm{~cm}$ (stations $57,58,59$ et 62 ) à $12 \mathrm{~cm}$ (stations 8,48 et 75 ) et, afin de traduire ce renseignement sur la carte, nous avons défini les trois catégories suivantes:

médiane inférieure à $6 \mathrm{~cm}$ : petits galets,

médiane comprise entre 6 et $8,4 \mathrm{~cm}:$ galets moyens,

médiane supérieure à $8,4 \mathrm{~cm}$ : gros galets.

Tous ces résultats ont été reportés sur la carte de la figure 3 et la vaste surface couverte par la sous-zone $I_{1}$ (environ de $1^{\circ}$ à $2^{\circ}$ de longitude ouest) souligne la pauvreté des fonds sédimentaires en graviers et en sables. Par ailleurs, les galets les plus gros se rencontrent dans cette sous-zone $I_{1}$ et notamment vers le sud-ouest où leur taille peut atteindre $15 \mathrm{~cm}$. Vers l'est, les galets deviennent plus petits et les cailloutis s'enrichissent en graviers et en sables. On y trouve successivement les trois sous-zones $I_{2}, I_{3}$ et $I_{1}$ et même la zone II. Dans ce secteur, notons qu'il s'agit surtout d'un accroissement de la teneur du sédiment en graviers et que la phase sableuse reste généralement faible. Elle ne dépasse en effet $2 \%$ que pour 7 stations et seuls les dragages $2.5,6$ et 25 en renferment plus de $5 \%$ (teneur exprimée par rapport au poids de sédiment total). Souvent cette fraction sableuse existe en très faible quantité; elle ne correspond alors qu'aux grains piégés par les organismes encroütants et libérés par nos diverses manipulations.

Cette modification des fonds d'ouest en est s'accompagne en outre d'un enrichissement en coquilles appartenant pour la plupart aux espèces suivantes (1) :
Modiolus barbatus,
Chlamys distorta,
Venerupis pulastra,
Glycymeris glycymeris,
Venus ovata,
Venus verrucosa
Chlamys varia,
Venerupis rhomboides,
Nucula nucleus,
Arca lactea,

\author{
Trivia arctica, \\ Buccinum undatum, \\ Ocenebra erinacea \\ Gibbula magus, \\ Emarginula fissura.
}

Dans les fractions galets et graviers de chaque dragage nous avons séparé et pesé coquilles entières et débris coquilliers, puis nous en avons établi le pourcentage par rapport au poids total de sédiment recueilli. Nos résultats ont été indiqués sur la carte de la figure 3 par l'intermédiaire de lettres dont la signification est la suivante : «c» de 2 à $5 \%$, «cc 》de 5 à $10 \%$ et «ccc》de 10 à $25 \%$. Ces proportions pondérales traduisent imparfaitement l'abondance des coquilles dans le sédiment. Exprimés en volume, ces résultats seraient approximativement à multiplier par 2,5 et donneraient une meilleure image de l'aspect des fonds.

Quant à l'épaisseur de la couverture sédimentaire, elle semble assez variable. Mince là où le substratum affleure: jurassique sur la bordure nord et sans doute éocène vers le centre, elle devient plus importante vers l'est où la drague ramène jusquà $50 \mathrm{~kg}$ de sédiment. La faune fixée sur les galets (Bryozoaires et Annélides polychètes notamment) est peu abondante, les graviers sont lisses et, compte tenu de la vigueur des courants, il est possible que certaines particules se déplacent encore : granules, grains de sable et débris coquilliers en particulier. Quelques Mollusques tels que : Venerupis rhomboides. Glycymeris glycymeris et Buccinum undatum peuplent ces fonds. Vers le sud-ouest, les cailloutis sont grossiers et les galets présentent généralement une face dépourvue d'organismes encroûtants, preuve de leur immobilité. Dans certains cas, après un travail extrēmement brutal, la drague ne ramène que quelques gros galets arrachés du fond. Les organismes ne recouvrent alors que la partie «supérieure» de ces cailloux; sables et graviers semblent inexistants et ces caractères suggèrent une sorte de pavage dont nous ne pouvons apprécier l'épaisseur.

(1) Déterminations de MM. F. Bénaro et J. Cléret, du Laboratoire maritime de Luc-sur-Mer, à qui nous adressons nos remerciements. 

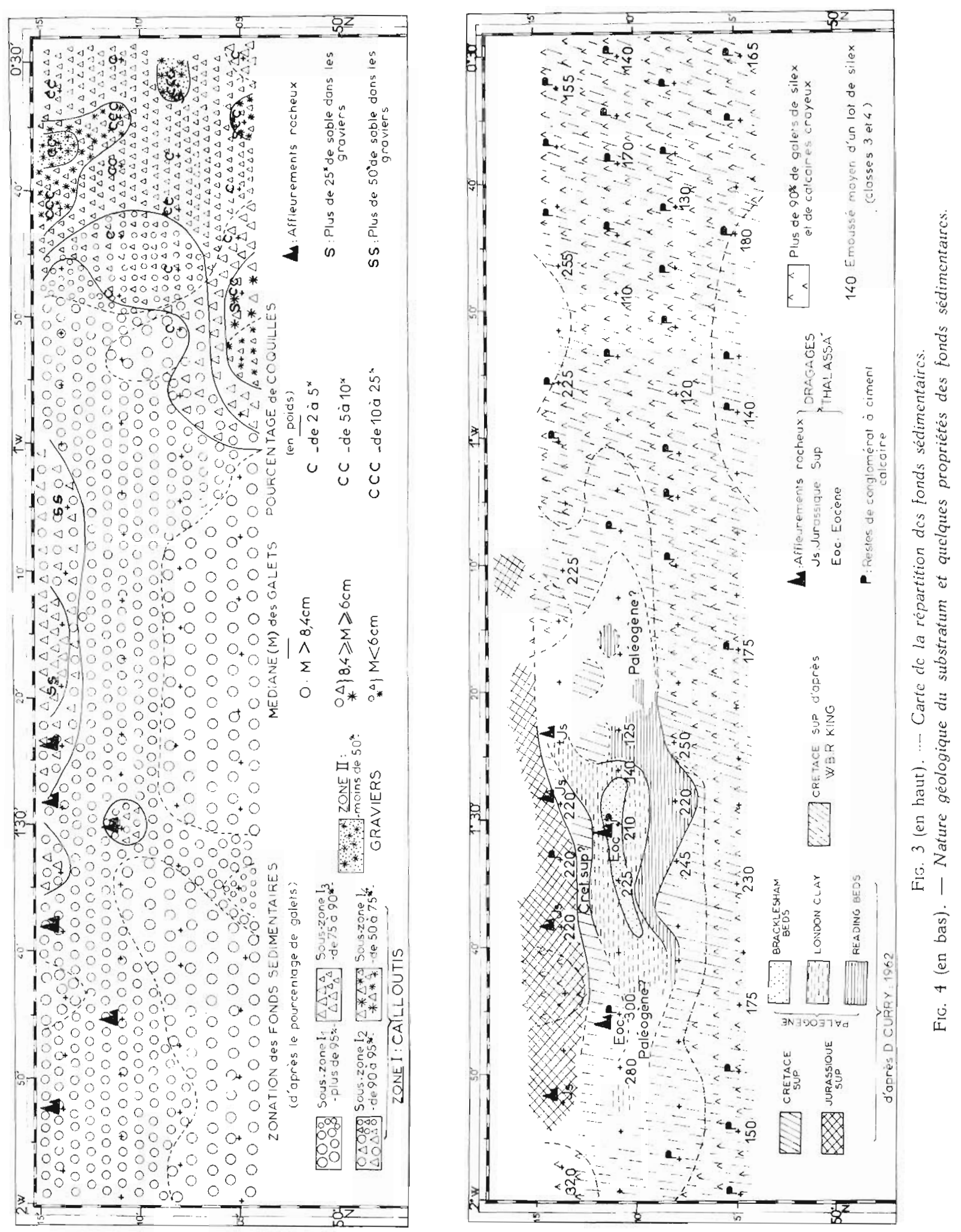
Enfin, comme G. Boll.tot (6) l'a noté en Manche occidentale, la faune de ces fonds à cailloutis est caractérisée par l'extraordinaire abondance des Ophiures. Elles sont surtout nombreuses vers le sud-est et plus précisément aux stations $4,5,8,10,23,25,28,56$ et 58 . Encore très abondantes aux stations 13, 37 et 70, elles sont plus rares vers le nord et vers l'ouest. La faune fixée est par contre plus abondante en ces régions qu'à l'est en raison de la plus grande stabilité des fonds. Il s'agit surtout d'Ascidies, d'Eponges, de Bryozoaires, d'Annélides polychètes sédentaires et plus rarement de balanes (station 43). Certaines stations sont encore remarquables par l'abondance des Mollusques: Modiolus barbatus aux stations 13 et 67, Chlamys varia et Chlamys distorta à la station 71 sur le substratum jurassique. Par l'importance de la faune libre ou fixée associée à ces fonds, cette région constitue une zone productrice de débris calcaire. C'est d'ailleurs là une des caractéristiques des fonds à cailloutis: G. Boll Lot (6).

\section{2. - Etude des galets.}

Dans cette région, ce qui frappe lorsque la drague déverse son contenu sur le pont du bateau, c'est l'abondance des silex. Ils représentent en effet plus de $80 \%$ de la fraction galets dans la moitié des dragages. Il s'y ajoute des fragments de roches variées dont il est intéressant de définir la nature et le pourcentage. On peut encore étudier la forme des galets et apprécier leur usure afin d'effectuer des comparaisons entre les diverses stations et éventuellement d'établir un rapport entre le substratum et la composition des galets en un point donné. Comme le caractère fondamental d'un galet est sa nature pétrographique, nous aborderons leur étude en fonction de ce critère.

\section{a) Silex.}

Ils sont surtout très abondants vers l'est et sur la bordure sud de la zone étudiée, mais il est rare qu'ils constituent moins de $50 \%$ de la fraction galets (stations 8, 17, 40,71, 72, 73 et 74). Généralement peu usés, ils sont de forme capricieuse et présentent un épais cortex blanchâtre de 0,5 à $1 \mathrm{~cm}$ environ. Ces silex proviennent du démantèlement de la craie et dans certains cas, il existe encore des vestiges crayeux ménagés par l'érosion (stations 2, 11,48 et 62). Ces restes témoignent d'un faible remaniement et indiquent que le substratum crétacé est proche. Si l'on se reporte aux recherches antérieures de W.B.R. KING (18)et D. Curry (11), ces stations sont effectivement situées sur la craie (voir carte fig. 4). En quelques endroits par contre, on recueille des silex très roulés, ovö̈des et présentant de nombreuses marques de chocs en « coups d'ongle 》 (stations 17 , $40,42,43$ et 47). Ces caractères attestent d'un important brassage, et il n'y a plus alors à chercher de relation entre ces silex et la nature du substratum. Pour L. Dangeard (12), ces galets de silex indiquent l'existence d'un ancien cordon littoral, et sa station la plus typique à cet égard est la 246 $\left(50^{\circ} 12^{\prime} \mathrm{N}\right.$ et $\left.2^{\circ} 2^{\circ} \mathrm{O}\right)$ située au nord-ouest de notre station 45 . Dans les mêmes parages, il signale encore la présence de ces galets à la station $396\left(50^{\circ} 10^{\prime} \mathrm{N}\right.$ et $\left.1^{\circ} 24^{\prime} \mathrm{O}\right)$, soit entre nos stations 37 et 38. Nous pouvons constater que les stations de L. DANGEARD et les nôtres, où les actions marines ont été particulièrement intenses, jalonnent une bande sensiblement est-ouest située entre $1^{\circ} 24^{\prime}$ et 2"2' de longitude ouest ce qui s'accorde avec l'hypothèse du cordon littoral ancien.

Afin de juger plus précisément des caractères morphoscopiques des silex, nous avons effectué des mesures d'indice d'émoussé sur des lots des classses 3 et 4 selon la méthode indiquée par A. Callleux (9). Pour chaque lot, nous avons défini graphiquement la valeur de l'indice d'émoussé moyen qui correspond à l'ordonnée 50 \% sur la courbe cumulative, et nous avons reporté les valeurs obtenues sur la carte de la figure 4. Le façonnement est d'autant plus marqué que ces valeurs sont élevées. Il apparait par conséquent que les silex situés dans les secteurs est et sud de la zone étudiée ont subi des actions érosives moindres que ceux des parties nord et ouest. Or, un façonnement atténué traduit un remaniement peu important et la présence d'un substratum crayeux sera d'autant plus probable que les valeurs de l'indice d'émoussé moyen seront faibles. Les tracés géologiques de W.B.R. King et de D. CuRry reportés sur la carte de la figure 4 confirment bien cette manière de voir. Cependant, pour les stations 15, 16, 18, 42,43 et 45, elles aussi situées sur la craie, nous avons obtenu des valeurs assez fortes qui témoignent d'un brassage important. Seulement, d'après ce que nous avons vu plus haut, il existe vraisemblablement dans le secteur ouest un ancien cordon littoral et les stations indiquées ci-dessus le soulignent. 
Notons enfin que la plupart des silex étudiés possèdent en surface une patine ferrugineuse rousse. L. Dangeard (12), qui a longuement étudié ce caractère, conclut à une origine sub-aérienne.

\section{b) Les calcaires crayeux, les craies blanches et les craies glauconieuses.}

Des galets crayeux de teinte beige accompagnent souvent les silex et ils abondent surtout aux stations $7,10,11,16,21,22,23,25,26,27,28,30,32,33,56,57$ et 59 où ils représentent plus de $10 \%$ de la fraction galets. Leurs formes sont généralement anguleuses et ils sont toujours profondément attaqués par les perforants : Annélides, Mollusques et surtout Cliones. Les fragments de craie blanche et friable sont extrêmement rares étant donné leur fragilité (stations 1, 11 et 23). En général, il s'agit de galets assez durs qui offrent l'aspect de véritables calcaires et qui sont constitués d'un ciment microcristallin qui devient parfois macrocristallin par recristallisation de la calcite. On y trouve en outre de nombreux débris d'organismes : fragments de Lamellibranches, de Bryozoaires, d'Echinodermes et d'Ostracodes. G. BiGnot (1) qui a examiné quelques plaques minces taillées dans ce matériel (galets des stations 8 et 30 ) note encore la présence de prismes d'Inocérames, de Foraminifères divers : Arénacés, Lagénidés, Rotalidés et Pélagiques (Gumbelina et Globotruncana) ainsi que Pithonella ovalis Kaufmann. Pour conclure, il attribue à ces galets crayeux un àge sénonien probable.

Les craies glauconieuses ou les calcaires gréseux glauconieux se rencontrent beaucoup plus rarement et existent toujours en très faible quantité ; les stations $25,38,60,64,65$ et 66 sont les plus remarquables à ce sujet. Tous les échantillons étudiés en plaque mince par G. Bignor peuvent être attribués au Crétacé supérieur.

Comme les silex et les galets crayeux proviennent du Crétacé supérieur, nous avons indiqué sur la carte de la figure 4 les zones où ils constituent ensemble plus de 90 \% $\%$ de la fraction galets. D'après les contours géologiques de W.B.R. KING et de D. Curry on constate qu'il existe une étroite correspondance entre ces zones et le substratum crayeux. En l'absence de roche en place, ce pourrait ètre là l'un des moyens permettant de juger de la nature du substratum. Ce critère serait d'autant plus valable que le pourcentage de galets crayeux serait fort et que les silex seraient peu façonnés. Il existe bien entendu des difficultés aux limites et notamment dans le secteur ouest où se relèvent quelques anomalies. En ce qui concerne les stations 5, 6, 7 et 8, leur fraction galets renferme un pourcentage élevé de roches éruptives ou sédimentaires anciennes dont l'origine est sans rapport avec le substratum.

\section{c) Les roches antécambriennes ou paléozö̈ques.}

Les types pétrographiques rencontrés sont extrêmement variés et nous les citerons par ordre d'abondance et de fréquence.

Roches peu abondantes et assez fréquentes.

Granites gris ou roses où les feldspaths sont souvent kaolinisés et les biotites chloritisées (46 stations).

Quartzites gris parfois noirs (28 stations)

Grès blonds ou rosés (31 stations)

Grès feldspathiques roses ou jaunâtres (30 stations)

Grès quartzeux (17 stations).

Roches très peu abondantes et peu fréquentes.

Microgranites à structure porphyrique de teinte gris rose (10 stations)

Quartz de couleurs très variées ( 8 stations).

Roches rares.

Granite à amphibole (4 stations)

Granite tectonisé à filonnets de quartz (5 stations)

Dolérite (4 stations)

Diorite quartzique (2 stations)

Grès psammitique (4 stations)

Roches d'origine métamorphique: schistes à minéraux (6 stations) et phyllades (2 stations).

(1) Nous adressons à G. Bıgnot, du Laboratoirc de Mi cropaléontologie de Paris, nos vifs remerciements pour les observations et déterminations qu'il nous a faites. 
Ces roches se rencontrent partout, mais elles abondent surtout aux stations 5, 7 et 8 et dans la partie médiane de la zone étudiée entre $1^{\circ}$ et $2^{\circ}$ de longitude ouest. Elles sont d'origine lointaine et leur gisement doit se situer dans le Massif armoricain ou dans le Devon et le Cornwall. Leur degré d'usure est variable, certains galets sont encore très anguleux alors que d'autres attestent d'un façonnement d'origine marine. Etant donné le nombre toujours faible de ces roches dans nos dragages, nous ne pouvons pas fournir de résultats morphométriques. Cependant, même si cela était possible, l'interprétation des résultats serait très difficile en raison de l'histoire complexe de ces galets. Comme le pense L. Dangeard) (12), certains ont pu être transportés par les glaces flottantes au cours du Quaternaire, mais d'autres sont peut-être dus à des apports fluviaux. De plus, au cours des transgressions, ils ont pu subir une action marine assez intense. C'est ainsi que là où il existe vraisemblablement un cordon littoral ancien, nous avons rencontré des éléments plus façonnés qu'ailleurs. Pour 10 galets de la station 39. nous avons en effet obtenu un indice d'émoussé moyen de 270.

\section{d) Les autres roches.}

Argiles ou marnes du Jurassique supérieur. Aux stations 71, 72, 74 et 77, la drague ramène des galets dont la nature est identique aux nombreux fragments arrachés au substratum en ces lieux et rapportés au Kimméridgien supérieur Portlandien inférieur (voir plus loin). De tels galets ont encore été recueillis aux stations 70 et 78 . soit toujours à proximité de leur gisement. La fragilité de ces éléments interdit en effet un long transport et ils constituent un excellent indice pour connaître la nature géologique des fonds.

Faciès ferrugineux divers. Aux stations 35, 37, 38, 42, 66, 68 et surtout 39, c'est-à-dire à proximité de la zone où D. Curry (11) a découvert divers niveaux appartenant à l'Eocène inférieur, la drague ramène des galets rougeâtres de nature variée: nodules argileux et ferrugineux, argiles rousses ferrugineuses, sableuses et glauconieuses, calcaires marneux légèrement ferrugineux, grès calcareux rougeâtres renfermant des débris de coquilles... Nous ne disposons d'aucun critère paléontologique pour dater ces éléments, mais certains de ces faciès ont été décrits par D. Curry dans l'Eocène inférieur et il est probable que nos galets en proviennent.

Enfin, la station 39 nous a livré 2 galets particulièrement intéressants dont les dimensions sont les suivantes: $120 \times 70 \times 18 \mathrm{~mm}$ et $140 \times 130 \times 25 \mathrm{~mm}$.

Le premier possède un noyau finement gréseux, de teinte beige, où s'observent des passées de glauconie et un cortex zoné de 7 à $8 \mathrm{~mm}$ d'épaisseur entièrement ferruginisé. L'une des faces présente des fentes de retrait polygonales de 2 à $3 \mathrm{~cm}$ de côté qui semblent postérieures à la formation du galet et qui n'affectent que le cortex. Sur la haute plage de Villers-sur-Mer, nous avons observé des galets de marnes oxfordiennes récemment façonnés par la mer et présentant des fentes de retrait d'un aspect assez semblable à celui que nous venons de décrire.

Le second galet, fortement patiné, est constitué d'une roche argilo-ferrugineuse légèrement calcaire. Il présente des perforations de Mollusques remplies d'un grès calcaire ferrugineux à débris de Lamellibranches et de Foraminifères: Rotalidés et Pélagiques sans plus de précision malheureu sement. Il n'est donc pas possible de dater ces différentes formations qui témoignent d'une histoire géologique complexe.

Etant donné que ces deux galets sont peu usés, que les éléments de roche analogue sont assez nombreux en cette station 39 et que nous y avons recueilli un fragment de grès calcaire ferrugineux en place et de nature voisine, il est permis de penser, d'après les contours géologiques de D. Curry. qu'ils sont issus de l'Eocène inférieur.

Meulières à Characées. Aux stations 39 et 72, nous avons récolté un galet assez anguleux de meulière renfermant dans un ciment calcédonieux, de petits Gastéropodes et de nombreuses tiges de Characées. Lâge de ces galets est difficile à préciser, mais nous savons que ces roches sont fréquentes dans le Tertiaire du bassin de Paris. Par ailleurs, nous les avons trouvés à proximité des gisements eocènes, ce qui indique que la série du Tertiaire est sans doute plus complète que celle qui a été mise en évidence. Ajoutons que des galets analogues ont été signalés par G. Boillot dans la fosse du «Pluteus» en 1963 (5). 


\section{3. - Etude des graviers et des sables.}

\section{a) Granulomélrie.}

Nous n'avons effectué une granulométrie complète de la fraction graviers et sables que pour les dragages où la teneur en particules inférieures à $2 \mathrm{~cm}$ était suffisamment importante, soit en général pour des sédiments appartenant à la zone II ou aux sous-zones $I_{3}$ et $I_{4}$. Dans les autres cas, nous n'avons fait qu'un seul fractionnement sur le tamis à mailles de $2 \mathrm{~mm}$ afin de séparer les graviers des sables.

La série de tamis utilisés est la suivante : $10 \mathrm{~mm}, 5,2,1,0.490,0,197,0,099$, et $0,049 \mathrm{~mm}$. Elle permet donc d'obtenir directement les principales parties constituantes du sédiment que sont: gros graviers, petits graviers, granules, sable grossier, sable moyen, sable fin et sable très fin (refus des 2 derniers tamis).

Ce fractionnement étant réalisé, on peut ensuite construire la courbe cumulative et définir la médiane. Celle-ci est généralement comprise entre 7 et $11 \mathrm{~mm}$, elle atteint même $15 \mathrm{~mm}$ pour la station 15 et ces valeurs soulignent la grossièreté de cette fraction inférieure à $2 \mathrm{~cm}$. Pour la station 2 , assez riche en sables $(19,9 \%$ du poids total de sédiment recueilli), la médiane plus faible est de $2.5 \mathrm{~mm}$. Elle n'est inférieure à $2 \mathrm{~mm}(1,3 \mathrm{~mm})$ que dans le cas particulier de la station 67 où la forte proportion de sable contenu dans les graviers est due au piégeage de grains de quartz et de débris coquilliers par les Modioles. Les sables se trouvent pris dans l'enchevêtrement des filaments du byssus et y adhèrent même grâce au mucus.

Les sédiments sont donc avant tout graveleux, et la partie sableuse et coquillière qui s'y ajoute est variable et dépend de la vigueur des courants. Il existe sans doute vers le sud-est de la zone étudiée un petit secteur plus calme étant donné que les sédiments qui s'y trouvent sont plus riches en particules fines: stations 2 et 25 notamment.

\section{b) Composition.}

Souvent, cette fraction inférieure à $2 \mathrm{~cm}$ apparaît essentiellement constituée de silex. Cependant. il arrive que les coquilles et les débris coquilliers soient très nombreux, et un examen plus approfondi montre qu'il existe en outre des fragments d'organismes divers, ainsi que des graviers de calcaires crayeux et de roches anciennes.

Sur tous nos échantillons, nous avons effectué une attaque à l'acide chlorhydrique afin de connaître le taux global de calcaire contenu dans cette partie du sédiment. Les résultats obtenus sont extrêmement variables et les teneurs en $\mathrm{CO}_{3} \mathrm{Ca}$ se situent entre 7 et $80 \%$ : stations 3 et 27 . Cette diversité est due en première approximation à la plus ou moins grande abondance de silex ou de coquilles.

Afin de préciser nos résultats et de juger de la répartition uniforme ou non du calcaire dans le sédiment, nous avons recherché la teneur en calcaire de chaque résidu de tamis. Pour cette étude, nous avons choisi 4 sédiments assez typiques provenant des stations $2,25,58$ et 61 . La teneur globale de ces échantillons en $\mathrm{CO}_{3} \mathrm{Ca}$ est respectivement de $: 36,35,8,66,9$ et $30,4 \%$.

A l'exception de l'échantillon de la station 58, la fraction insoluble est la plus importante et c'est elle qui constitue l'essentiel des graviers (de 70 à $80 \%$ du refus des tamis 5 et $10 \mathrm{~mm}$ ). Puis le taux de calcaire augmente, il est de 45 à $50 \%$ dans les granules et il atteint $70 \%$ dans le sable grossier. C'est généralement cette fraction la plus riche en $\mathrm{CO}_{3} \mathrm{Ca}$, car le sédiment s'enrichit ensuite en éléments détritiques: il n'y a plus que 20 à $25 \%$ de calcaire dans le sable fin. Pour la station 58 , la courbe du calcaire est sensiblement identique, mais le sédiment est très riche en coquilles, ce qui entraine un taux de calcaire beaucoup plus fort dans les graviers, $65 \%$, et surtout dans les grariules, $90 \%$.

Ces résultats montrent que la distribution du calcaire dans les différentes classes granulométriques n'est pas analogue à celle des éléments siliceux. En conséquence, la teneur en CO. Ca est d'autant plus forte que le pourcentage en particules comprises entre 0,5 et $5 \mathrm{~mm}$ est élevé. Par contre elle est faible lorsque les gros graviers sont abondants: station 3 par exemple. Il faut cependant noter, comme nous l'avons vu pour la station 58, que la présence de nombreuses coquilles dans le refus des tamis 5 et $10 \mathrm{~mm}$ mène à des résultats différents. C'est pour cette raison que les 
échantillons des stations 27,29 et 56 renferment plus de $60 \%$ de $\mathrm{CO}_{3} \mathrm{Ca}$, respectivement : 75,8, 63,5 et 68,3 . Enfin, le calcaire détritique contenu dans nos échantillons n'a généralement que peu d'influence sur le taux global de $\mathrm{CO}_{3} \mathrm{Ca}$ obtenu. Néanmoins, pour l'échantillon de la station 61 il existe $17 \%$ de calcaire détritique pour un taux global de $30.4 \%$. Il est donc indispensable dans ce cas de tenir compte de cette fraction pour juger de l'importance exacte de la partie organogène. Le calcaire détritique est essentiellement composé de graviers de calcaires crayeux et se rattache, par son origine, à la partie résiduelle insoluble du sédiment.

Cette remarque nous conduit à rechercher la nature exacte des constituants de cette fraction inférieure à $2 \mathrm{~cm}$ ainsi que leur répartition dans les différentes classes granulométriques. Pour cette étude, nous avons repris les 4 échantillons précédents et nous y avons ajouté ceux des stations 5, 67 et 70 dont les teneurs globales en $\mathrm{CO}_{3} \mathrm{Ca}$ sont respectivement de $32,8,44,2$ et $48 \%$. Pour chaque refus de tamis nous avons déterminé les éléments en présence et nous en avons évalué le taux par comptage. La fraction détritique est essentiellement formée de quartz pour la phase sableuse, et de graviers de silex et de roches anciennes. La fraction organogène renferme surtout des coquilles et des débris coquilliers auxquels viennent s'ajouter des pièces de Balanes, des fragments de Bryozoaires et des restes d'Ophiures : plaques brachiales, « vertèbres » et spicules.

Sur les graphiques des figures 5 et 6 , nous montrons les courbes de la répartition granulométrique de tous ces éléments. Les silex sont généralement très abondants dans les graviers. leur pourcentage diminue ensuite rapidement et ils deviennent rares dans les sables grossiers. Les calcaires détritiques se répartissent équitablement dans les graviers alors que les éléments de roches anciennes (granites et surtout quartzites, grès quartzeux et grès) se rencontrent essentiellement dans les granules où ils constituent souvent la fraction la plus importante de la partie insoluble. Dans la phase sableusc, la teneur en quartz n'est vraiment importante que dans les sables moyens et fins. En ce qui concerne les débris organogènes, il apparaît que les pièces de Balanes se trouvent surtout dans les granules et les sables grossiers tandis que les débris d'Ophiures n'abondent que dans les parties fines du sédiment. Nous n avons pas obtenu de résultats aussi nets pour les fragments de Bryozoaires, mais ceux-ci intéressent diverses espèces, et, pour chacune d'elles il existe une répartition beaucoup plus limitée. C'est un peu ce que l'on retrouve pour les débris coquilliers qui eux aussi appartiennent à des espèces diverses. De plus, l'existence de coquilles entières dans les graviers entraîne des diff́́rences importantes d'une station à une autre. Quoiqu'il en soit, pour tous les échantillons étudiés nous avons relevé une grande abondance de débris coquilliers dans les granules et les sables grossiers. Si l'on ajoute à cela que les pièces de Balanes et les débris de Bryozoaires sont fréquents aux mêmes dimensions, on conçoit aisément que ces deux fractions soient souvent les plus riches en $\mathrm{CO}_{3} \mathrm{Ca}$.

Grâce à ces résultats on constate que la nature des éléments organogènes peut modifier le taux de calcaire dans les différentes classes granulométriques. De plus, ceux-ci ne sont généralement pas roulés et se rencontrent à proximité des lieux où vivent les espèces correspondantes. La faune qui vit sur les fonds a donc une influence directe sur la répartition et le taux de calcaire organogène dans le sédiment.

Enfin, pour compléter les résultats obtenus, nous avons construit pour les échantillons des stations 2, 25, 58 et 61 les courbes de fréquence (abscisses logarithmiques et ordonnées arithmétiques) selon une méthode utilisée par P. Hommeril (16). Sur un même graphique (fig. 7), nous avons tracé la courbe de la fraction calcaire organogène, celle de la fraction détritique (y compris le calcaire détritique) et celle du sédiment entier. Les pourcentages sont exprimés en fonction du résidu entier, de telle sorte que la courbe du sédiment entier représente la somme des deux autres.

Pour l'échantillon de la station 2 , le plus riche en sables $(19,9 \%)$, la courbe du calcaire organogène est très différente de celle de la fraction détritique. Les maximums ne se correspondent pas et le sédiment entier est très dispersé. Peut-être cette divergence est-elle due à la nature même des èléments organogènes essentiellement constitués de débris coquilliers, lesquels abondent entre 0,5 et $5 \mathrm{~mm}$. Cependant, il semble bien que cela traduise un brassage insuffisant de cette fraction fine du sédiment. La partie graveleuse détritique n'évolue guère actuellement et conserve des caractères acquis anciennement. Elle constitue avec les galets la phase caillouteuse résiduelle. Par contre. les débris organogènes et le quartz sont soumis à l'action des courants et acquièrent un état d'équilibre fonction des conditions actuelles. Les deux fractions se superposent sans s'accorder. Pour les échan- 

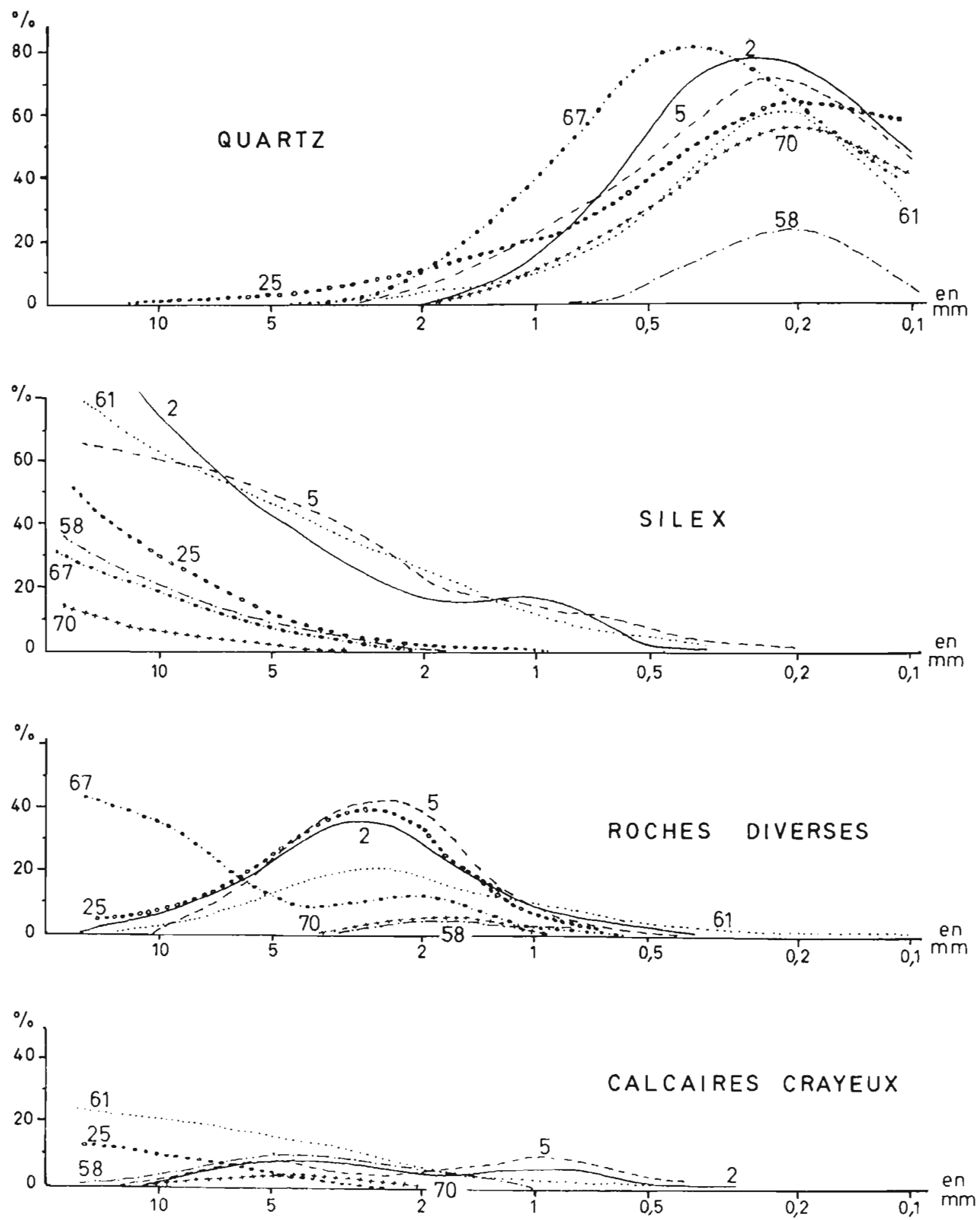

FIG. 5. - Répartition granulométrique des éléments dêtritiques dans les graviers et les sables des stations 2, 5, 25, 58,61, 67 et 70 . 

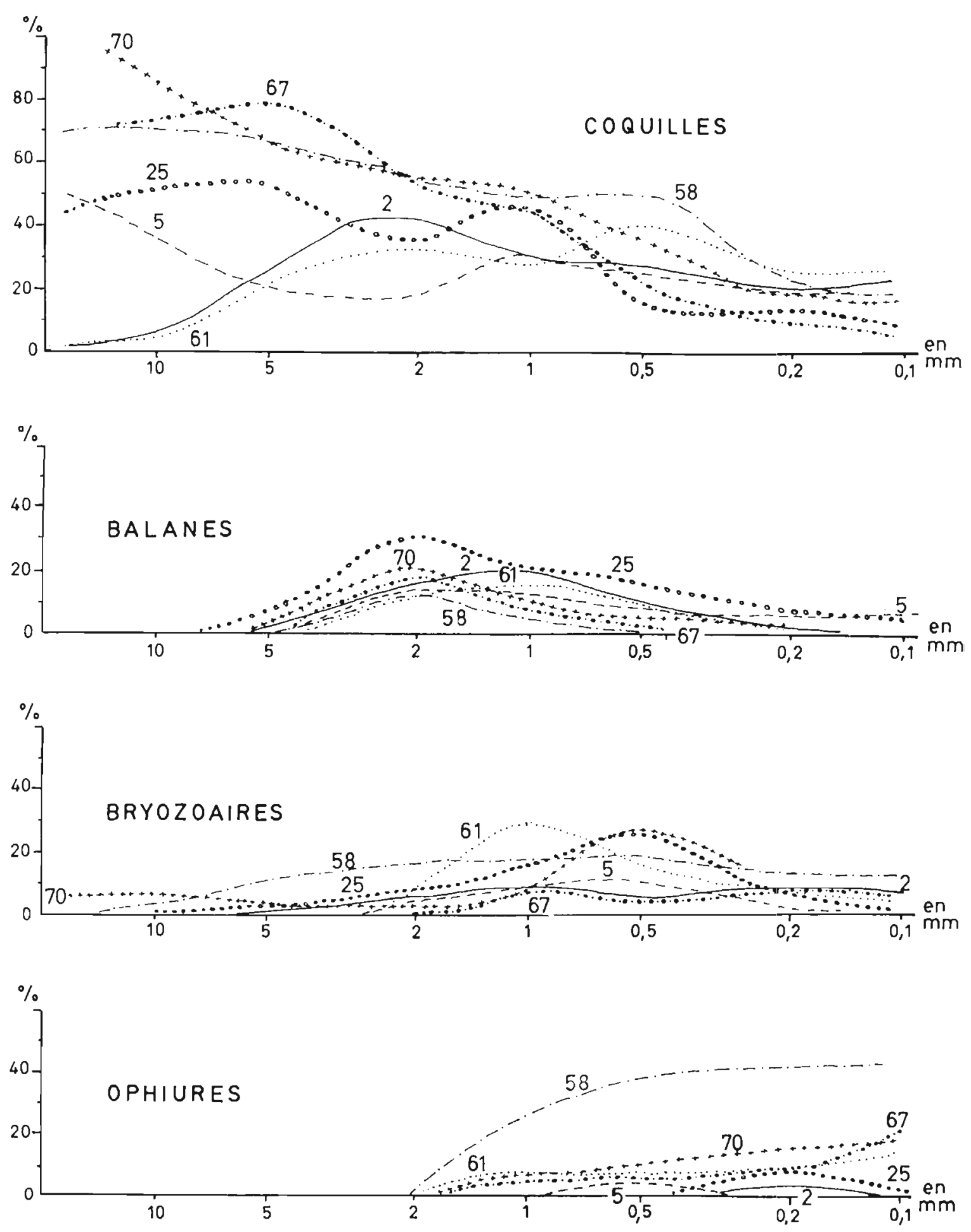

FIG. 6. - Répartition granulométrique des débris organogènes dans les graviers et les sables des stations 2. 5, 25, 58,61,67 et 70 . 
tillons des stations 25.58 et 61 , le calcaire organogène est surtout important dans les graviers, la courbe est généralement assez étalée et le triage est moins bon que pour la partie détritique. Ce caractère peut s'expliquer comme précédemment, mais les résultats obtenus pour ces échantillons ne sont pas aussi nets que ceux de la station 2. Il est vrai qu ils sont très pauvres en sables et qu'il est par conséquent difficile de juger de la répartition des particules fines dans le sédiment.
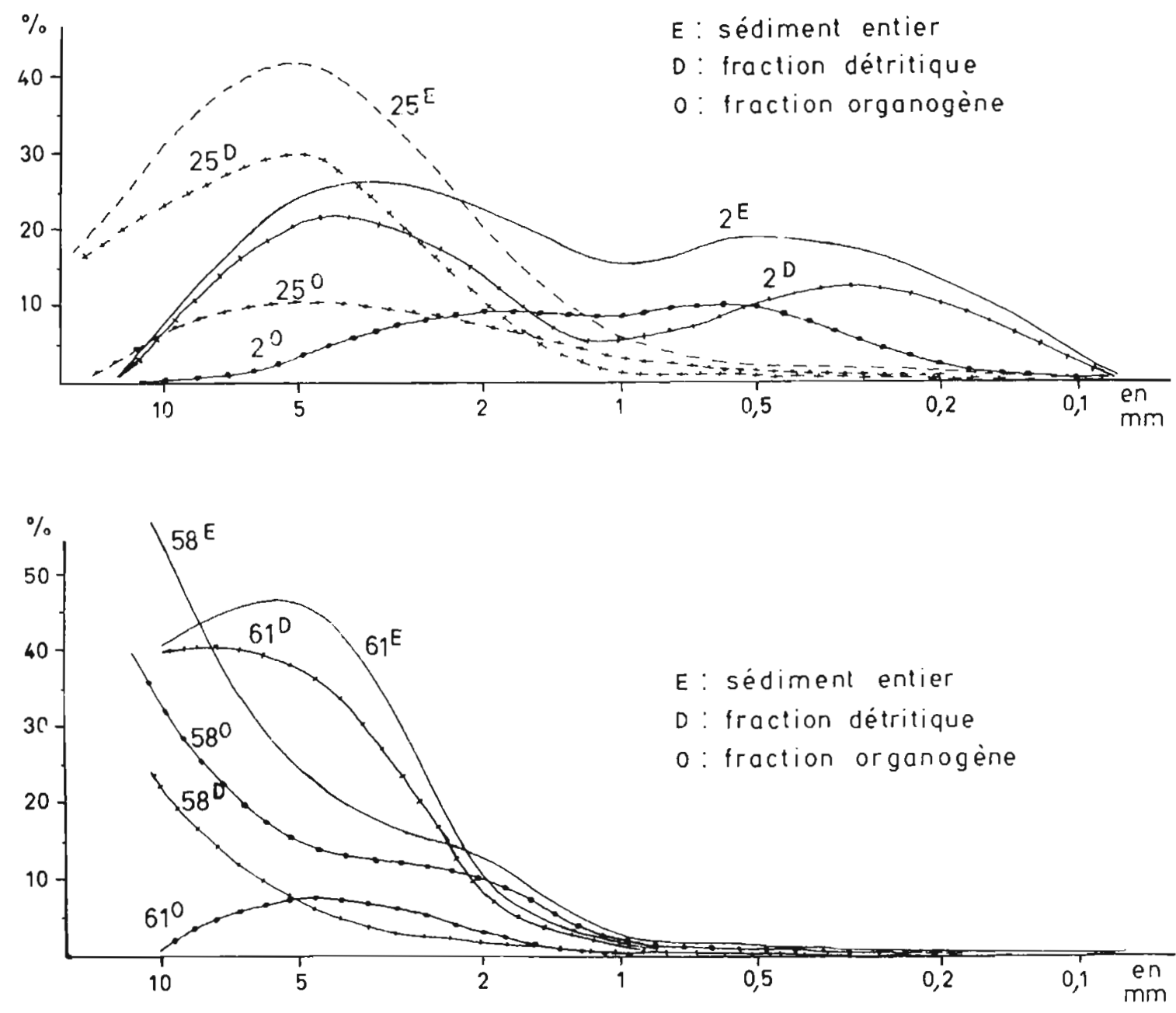

FIG. 7. - Courbes de fréquence pour les graviers et les sables des stations 2, 25, 58 et 61 .

\section{c) Morphoscopie des grains de quartz.}

L'étude morphoscopique des grains de quartz a porté sur le résidu du tamis $0,490 \mathrm{~mm}$, et a été réalisée pour les sables des 15 stations suivantes: $2,3,5,6,11,25,26,39,49,57,58,61,62,67$ et 70 .

Les principaux résultats obtenus peuvent être résumés comme suit:

la proportion de grains non-usés est toujours faible: de 2 à $7 \%$;

les grains typiquement luisants sont rares;

il existe d'assez nombreux grains entièrement mats : sub-émoussés et émoussés notamment:

de nombreux quartz, encore mats, présentent un aspect lustré et d'autres montrent des surfaces luisantes entourant des plages plus ou moins picotées; ce picotis s'estompe progressivement aux bords de la surface mate;

certains grains à surfaces luisantes portent des marques de chocs en «coups d'ongle». 
Compte tenu de l'abondance des grains partiellement ou entièrement mats, il nous faut penser à une érosion d'origine éolienne. Cependant, l'aspect luisant d'un bon nombre de grains témoigne d'un façonnement dans l'eau. L'examen des quartz mats lustrés ou luisants montre que ce façonnement est postérieur à l'action du vent. Or, d'après l'histoire géologique de la Manche, G. Borllot, 1964 (6), nous savons que cette région était émergée pendant les régressions du Quaternaire. Il y régnait alors un climat périglaciaire et l'érosion éolienne y était intense. C'est dans ces conditions que les grains de quartz ont acquis leur aspect picoté mat. Ces grains ont été repris par la mer au cours des transgressions et notamment au cours de la transgression flandrienne. Ils ont alors subi l'action de l'eau et ont acquis un aspect voisin de celui que nous leur connaissons. Là où les courants sont sensibles sur le fond, cette action se prolonge encore actuellement, et elle est d'autant plus marquée que ces courants sont forts. C'est ainsi que les pourcentages de grains émoussés luisants et de grains mats lustrés ou luisants sont plus importants aux stations 49,67 et 70 qu'aux stations situées à l'est où l'abondance des débris coquilliers et sableux témoignent de zones localement plus calmes.

\section{Données géologiques}

Comme pour toute la Mer de la Manche, nous trouvons d'importants renseignements d'ordre géologique dans les travaux fondamentaux de L. Dangeard, 1928 (12) et de W. B. R. KING, 1954 (18). Par ailleurs, une partie de cette zone a été récemment étudiée par D. Curry, 1962 (11) et ses résultats sont parmi les plus précis que nous ayons en Manche. A partir de ces documents nous avons reporté les grandes lignes de la structure géologique de cette zone sur la carte de la figure 4.

Nos moyens d'investigation ne permettent pas une étude détaillée du substratum; cependant, quand la roche affleure, la drague arrache parfois quelques fragments dont l'étude peut apporter d'intéressants renseignements. De plus, l'étude des sćdiments fournit en ce sens de précieux résultats. Nous y avons trouvé, en particulier, des fragments d'un poudingue à ciment calcaire dont l'étude permet de préciser l'histoire géologique de cette région au Quaternaire. En outre, nous avons vu précédemment que l'examen des galets donne de précieux indices sur la nature géologique du substratum.

\section{1. - Dragages géologiques.}

\section{a) Jurassique supérieur.}

Dans la partie nord-ouest de la zone étudiée, 4 dragages ont fourni des morceaux argileux ou marneux, grisâtres, appartenant au Jurassique supérieur. Ces fragments sont perforés par des Mollusques (Hiatella sp. et Barnea parva), ils présentent une faune fixée abondante et se débitent facilement. Les positions de ces dragages, effectués le 18-IX-63, sont les suivantes:

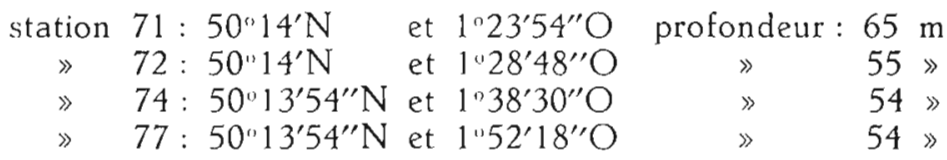

Ce matériel a été étudié par M. Rioult (1) qui a obtenu les résultats suivants:

Station 77. Argiles grises, très légèrement bitumineuses, à tests d'Ammonites nacrées et à débris de lignite. On y trouve : Astarte sp.. Astarte cf. mysis 0'Or8., Protocardia sp., des Ostracodes et des écailles de type cycloïde. Quant aux fragments d'Ammonites, il s'agit d'Aulacostephanus du groupe A. eudoxus D'OrB. Ces argiles appartiennent à la zone à Aulacostephanus eudoxus du Kimméridgien supérieur.

(1) Pour cette étude, et les conclusions très précises quil mạ apportées, je remercie vivement M. Rroult. du Labọatoire de Géologie de Caen. 
Station 71. Argiles grises, calcareuses, légèrement bitumineuses et à débris de lignite. Elles renferment des écailles de type cycloïde, des empreintes de Lamellibranches et d'Ammonites et Discina latissima J. Sow.

Ces argiles présentent beaucoup d'affinités avec celles de la station 77 .

Station 72. Les fragments recueillis se rapportent à 3 faciès distincts:

calcaires argileux à oolithes phylliteuses et phosphatées;

dolomies calcareuses à foraminifères pyriteux: Ophtalmidium et Cornuspira et à débris de Lamellibranches et d'Ammonites;

argiles noires, feuilletées, bitumeuses et à morceaux de lignite; on y trouve des restes de Foramini-

fères et d'Ostracodes, des débris d'Ammonites et Astarte cf. ingenua Lor.

Ces faciès se rattachent au passage Kimméridgien-Portlandien mais les deux premiers, pourtant très caractéristiques. ne semblent pas avoir d'équivalents sur le continent.

Station 74 . On y distingue 2 faciès assez voisins:

argiles feuilletées très bitumineuses, brunâtres à débris de lignite et de poissons (faciès schistes-carton); elles renferment des Ostracodes à test corrodé, des Foraminifères: Lenticulina, Astacolus et Spirillina, des moules ou des empreintes de Lamellibranches; Astarte sp., Lucina minuscula BlakE, Protocardia sp., un fragment d'Ammonite du type Subplanites sp. et de nombreuses plaques radiales pyriteuses du Crinoïde pélagique : Saccocoma sp.;

argiles noires, feuilletées, bitumineuses, à débris de poissons; les fossiles reconnus sont les suivants:

Lucina minuscula Blake, Astarte cf. mysis D'Orb., Discina latissima J. Sow. et des plaques radiales de Saccocoma sp.

Ces argiles se rattachent au faciès «Oil Shales»: zone à Subplanites sp. du Portlandien inférieur. Saccocoma sp. est en effet un fossile très caractéristique de cette zone.

Les différents faciès recueillis dans nos dragages jalonnent donc la limite Kimméridgien supérieur-Portlandien inférieur, de part et d'autre des couches repères à Gravesia.

Dans ces parages, les études de D. Curry (11) ont porté sur plusieurs stations et les faciès décrits sont voisins des nôtres: essentiellement des argiles sombres, plus ou moins indurées, parfois fossilifères. C'est ainsi qu'à la station 306, sensiblement située entre nos stations 77 et 78 , les argiles contiennent Aulacostephanus sp. Nous avons trouvé cette Ammonite à la station 77 dans des argiles grises; les résultats sont donc en parfait accord. Ailleurs, les fossiles sont moins abondants ou moins bien conservés, et D. CurRY conclut généralement Kimméridgien, parfois Kimméridgien supérieur; nos résultats précisent donc l'âge des formations jurassiques qui affleurent dans ce secteur. Dans un proche avenir nous nous proposons de poursuivre nos recherches dans cette région afin de mieux définir les contours géologiques et de mieux connaître la nature des contacts. D'après la cartographie de D. CuRry, les stations 71 et 72 sont en effet situées en dehors du Jurassique supérieur, or nous disposons actuellement de trop peu de stations pour proposer un nouveau tracé.

\section{b) Eocène inférieur (?).}

Aux stations 39 et 42, nous avons recueilli, en place, des fragments gréseux et argileux rougeâtres que nous n'avons pu dater avec prècision. C'est en raison de la cartographie de D. Curry, et des faciès voisins qu'il a décrits, que nous les attribuons à l'Eocène inférieur. Les positions de ces 2 stations sont les suivantes:

$$
\begin{aligned}
& \text { station 39: } 50^{\prime \prime} 11^{\prime} \mathrm{N} \text { et } 1^{\prime \prime} 30^{\prime} 24^{\prime \prime} \mathrm{O} \text { profondeur: } 76 \mathrm{~m}
\end{aligned}
$$

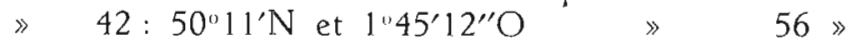

A la station 39, il s'agit d'un grès rougeâtre, calcaire et ferrugineux, qui renferme en outre des grains de glauconie et des débris coquilliers non identifiables. Nous avons vu qu'à cette station la drague ramène plusieurs galets de nature voisine.

A la station 42, ce sont de petits morceaux d'argile rousse, devenant localement plus sableuse, et riche en grains de glauconie. 


\section{2. - Fragments de poudingue à ciment calcaire.}

Dans de nombreux dragages nous avons récolté des fragments plus ou moins importants d'un poudingue à ciment calcaire et à graviers de silex ou de calcaires crayeux. Les éléments de roches anciennes y sont plus rares et le ciment, très dur, contient des grains de quartz et parfois des débris coquilliers. Dans certains cas, i] s'agit de coquilles entières liées entre elles par un ciment calcaire, et, aux stations 34, 57 et 58, nous avons recueilli des échantillons particulièrement caractéristiques à ce sujet. Il arrive aussi qu'il n'existe plus qu'un vestige de ciment calcaire à la surface d'un galet : silex ou calcaire crayeux.

Plusieurs échantillons analogues ont été recueillis en Manche occidentale et notamment par G. Boillot ( 4 et 6 ) au large de Roscoff et de l'ile de Bréhat, mais il ne semble pas qu'ils soient aussi fréquents que dans la zone étudiée.

Les coquilles conservées dans les échantillons des stations 34, 57 et 58 appartiennent pour la plupart aux espèces suivantes: Chlamys varia, Buccinum undatum (jeune variété littoralis?), Venerupis (Tapes) rhomboides, Glycymeris glycymeris, Gibbula sp. (1).

Il convient cependant de noter que l'exemplaire du genre Glycymeris possède une coquille plus inéquilatérale que les Glycymeris glycymeris actuels, et que l'individu rapporté à l'espèce Venerupis (Tapes) thomboìdes est une variété plus grande, à test plus épais et à bord anal un peu moins tronqué que les spécimens actuels. G. Bolllot (6) a fait une observation analogue pour un individu du genre Glycymeris, et. bien que les formes décrites se retrouvent toutes actuellement en Manche, certains individus possèdent tout de même des caractères singuliers qui leur donnent un cachet ancien. Quoiqu'il en soit, ces poudingues ne renferment pas de fossiles caractéristiques et ils sont difficiles à dater.

Pour G. Boillot, la cimentation s'est produite à l'air libre, lors de la régression qui a suivi le dépôt d'un calcaire coquillier lié à la transgression tyrrhénienne.

Récemment, L. Dangeard et P. Hommeril (14) ont décrit des plaquettes de grès calcaire recueillies à Bernières-sur-Mer (Calvados) et datées par le carbone 14:23300 \pm 1000 ans. Pour ces auteurs : "le sable coquilier du banc consolidé s'est donc déposé sur le rivage de la mer basnormanienne (13)... La consolidation s'est faite, non en milieu marin, mais en milieu sub-aérien au cours d'un épisode d'émersion... Sans doute le phénomène s'est-il produit à la fin de l'époque würmienne... mais il ne faut pas exclure totalement la possibilité d'une consolidation pendant la régression pré-flandrienne».

Pour conclure, nous ne disposons pas de données d'âge absolu ni de fossiles caractéristiques; toutefois, nous pouvons penser que la plupart des consolidations par ciment calcaire connues en Manche ou sur son littoral, se sont sensiblement produites à la même époque, soit au début ou plutôt à la fin de l'époque würmienne. On recueille en effet en dragage des galets qui ont éclaté sous l'action du gel et qui sont recimentés par un ciment calcaire analogue à celui des poudingues. De tels galets ont été décrits par G. Boillor (6) et nous en avons recueilli un exemplaire particulièrement démonstratif à la station 55 .

Il en résulte que la mise en place des éléments organogènes d'origine marine, cimentés avec des grains de quartz, des graviers et parfois des galets, s'est produite lors de la transgression antérieure à la dernière glaciation : soit dans nos régiens au bas-Normannien (13).

Les fragments recueillis actuellement en dragage ou parfois rejetés à la côte, M. Rioult (19), proviennent du remaniement actuel et surtout flandrien de formations sans doute plus étendues comme en témoignent les vestiges de ciment calcaire conservés à la surface de certains galets. Cependant, il ne semble pas que ce remaniement ait été très important car les échantillons recueillis sont généralement anguleux et certaines coquilles, prises dans le poudingue, sont dans un état de conservation parfaite (aucune usure et teintes conservées). Par ailleurs, la résistance du ciment rend ces roches très solides et il est difficile d'imaginer un substratum de cette nature entièrement démantelé et réduit aux graviers et aux galets qui en étaient les constituants. Dans cette hypothèse, les coquilles ne seraient pas aussi bien conservées et les calcaires crayeux auraient

(1) Déterminations et observations de MM. BÉnARd et Ctéret, du Laboratoire maritime de Luc-sur-Mer, et FISCHER du Muséum national d'Histoire naturelle. 
sans doute disparu. Enfin, la forme souvent capricieuse de la plupart des fragments et la présence de vides au centre de certains semblent bien indiquer une cimentation partielle et très irrégulière.

Nous pensons en effet que les phénomènes de consolidation par ciment calcaire ont pris naissance localement, grâce à la circulation dans un sédiment sablo-graveleux (parfois caillouteux) coquillier, d'eaux bicarbonatées qui ont ensuite laissé précipiter le carbonate de calcium. Selon la granulométrie du sédiment imprégné et la richesse des eaux en bicarbonate de calcium, on obtenait des zones consolidées plus ou moins étendues et ramifiées. Le bicarbonate de calcium pouvait provenir de la dissolution de la fraction organogène contenue dans le sédiment. Mais, avant de circuler dans celui-ci, et là où le sol est crayeux, les eaux pouvaient être très chargées en calcaire. Ajoutons que dans de telles régions, le sédiment devait contenir une importante fraction de calcaire détritique, elle aussi susceptible d'être mise en solution. La présence d'un tel substratum favorisait la formation de niveaux consolidés et c'est là qu'ils devaient être les plus étendus. Comme l'indique la carte de la figure 4 pour la zone étudiée, c'est effectivement sur le Crétacé supérieur crayeux que se rencontrent les témoins les plus fréquents de ces cimentations quaternaires (sur 31 stations qui en ont fourni des restes, 28 sont situés sur la craie).

Parallèlement, nous pouvons constater que les terrasses normaniennes sont fréquentes sur le littoral normand, mais qu'il est rare quelles renferment des plaquettes gréso-calcaires. Celles-ci ne sont connues qu'à Bernières-sur-Mer et, plus à l'ouest, à Saint-Côme-de-Fresné : Guillaume 1948 (15). Or, dans ces deux localités, le substratum bathonien est calcaire. De plus, les cimentations observées n'affectent toujours, dans les sables normanniens, qu'un mince niveau dont les parties inférieures et supérieures sont irrégulières. Cet aspect témoigne d'un phénomène local et peu étendu identique à ce que nous proposons pour les consolidations connues au fond de la Manche.

\section{Conclusion}

La région étudiée est donc presque entièrement occupée par des cailloutis, mais la distinction de plusieurs sous-zones nous a permis de la subdiviser. Vers l'ouest, là où la roche n'est pas à nú, les fonds sont couverts de gros galets et la faune fixée s'y développe abondamment en raison de la présence de supports stables. De tels supports n'existent pas vers l'est où les cailloutis deviennent de plus en plus graveleux et forment une épaisse couverture sédimentaire qui masque le substratum.

Les courants sont partout assez forts, mais l'abondance de particules fines et de débris organogènes dans certains dragages du secteur sud-est témoigne de zones localement plus calmes. A la partie détritique et résiduelle du sédiment, composée de galets et de graviers essentiellement siliceux, vient alors s'ajouter une fraction calcaire organogène à laquelle se mêlent quelques grains de quartz remaniés. Il semble bien, comme nous l'avons vu pour notre échantillon le plus sableux issu de la station 2, que le brassage actuel n'affecte que cette seconde partie du sédiment. Comme celle-ci reste toujours très faible dans la zone étudiée, l'essentiel de la couverture sédimentaire est hérité de l'histoire géologique de la Manche au Quaternaire.

De nombreux éléments sont encore là pour nous aider à comprendre cette histoire complexe : abondance de galets de roches éruptives ou sédimentaires anciennes, émoussé très fort ou patine de certains galets, cailloux sans doute éclatés sous l'action du gel, morphoscopie des grains de quartz et poudingues à ciment calcaire. Il est difficile de saisir ce qui est antérieur à la transgression normannienne et il n'existe pas dans la zone étudiée l'équivalent de la nappe des cailloutis décrite par G. Bolllot en Manche occidentale (6). De l'époque normannienne date le dépôt de débris organogènes pris dans les poudingues à ciment calcaire. Pendant la régression pré-flandrienne cette région était émergée et soumise à un climat périglaciaire: les grains de quartz ont alors acquis un aspect mat et certaines roches se sont fragmentées ou patinées. Les cimentations calcaires datent sans doute de la fin de cette période et au Flandrien, la mer a de nouveau envahi cette zone y produisant divers remaniements et y laissant les traces d'une intense érosion marine : fort émoussé des galets, marques de chocs et aspect lustré ou luisant des grains de quartz notamment. Tous ces caraçtères n'ont guère été modifiés depuis. 
L'étude des sédiments et tout particulièrement celle des galets nous a permis de souligner l'étroite correspondance qui existe souvent entre le substratum et leur nature pétrographique. Ceci est très net sur la craie où l'ensemble formé par les silex et les calcaires crayeux constitue généralement plus de $90 \%$ de la fraction galets. C'est aussi vrai sur le Jurassique supérieur et l'étude minutieuse de ces éléments peut donc donner de précieux indices concernant la nature géologique du substratum.

Enfin, les quelques dragages géologiques réussis sur le Jurassique supérieur de la bordure nord de la zone étudiée, nous ont permis de préciser qu'il s'agissait là de faciès appartenant au Kimméridgien supérieur ou au Portlandien inférieur. De plus, nos résultats nous amènent à envisager une légère modification des contours géologiques dans ce secteur.

Les enseignements tirés de cette campagne en Manche orientale à bord de la « Thalassa» sont donc variés et très intéressants. Grâce à notre méthode de prospection systématique des fonds nous avons pu fournir une carte de la répartition des sédiments et aborder quelques problèmes d'ordre géologique. Nous nous proposons maintenant de reprendre en détail certains aspects de cette étude et de l'élargir.

\title{
Laboratoire de Géologie marine de Caen.
}

\author{
BIBLIOGRAPHIE
}

1. Berthols (L. ct C.), 1955. -- Etude lithologique des sédiments récoltés par le «Président-Théodore-Tissier» en Rade de Brest et en Manche (campagne 1949). - Rev. Trav. Inst. Pêches marit., I9 (4), p. 467-499.

2. Berthols (L.) et Furnestin (J.). 1938. - Etude des sédiments dragués par le « Président-Théodore-Tissier 》, 6" croisière. - Rev. Trav. Inst. Pèches marit., 11 (3), p. 381-424.

3. Bolllot (G.), 1960. - La répartition des fonds sous marins au large de Roscoff (Finistère). - Cah. Biol. mar.. 1. p. $3-23$.

4. - 1961. - La répartition des fonds sous-marins en Manche occidentale. - lbid., 2, p. 187-208.

5. - 1963. - Sur une nouvelle fosse dans la Manche occidentale: la fosse du «Pluteus». - C. R. Acad. Sci., Paris, 257, p. $3448-51$.

6. - 1964. - Géologie de la Manche occidentale: fonds rocheux, dépôts quaternaires, sédiments actuels. Ann. Inst. océanogr., 42 (1), 220 p.

7. Bolurcart (J.). 1941. - Essai d'une classification raisonnée des matériaux meubles. -- Bull. Soc. géol. France, 5" sér., p. 117-153.

8. - 1955. - Etude des échantillons récoltés par le «Président-Théodore-Tissier» en juillet-aoùt 1946. - Rev. Trav. Inst. Péches marit.. 19 (4), p. 447-464.

9. Callleux (A.), 1947. - L'indice d’èmoussé : définition et premiere application. - C. R. somm. Soc. géol. France. 10 nlov., p. $251-252$.

10. - 1954. - Limites dimensionnelles et noms des fractions granulométriques. - Bull. Soc. géol. France, 6 sér.. 4. p. $643-646$.

11. Curry (D.), 1962. - A lower tertiairy outlier in the central english channel, with notes on the beds surrounding it. -- Quart. J. geol. Soc. Londres, 178, part. 2. p. 177-205.

12. Dangeard (L.), 1928. - Observations de géologie sous-marine et d'océanographie relatives à la Manche. - Ann. Inst. océanogr., n. S., 6 (1), 296 p.

13. Dangeard (L.) et Graindor' (M. J.). 1956. - T.étage normannien et ses divisions. - C. R. Acad. Sci.. Paris, 242. p. $1744-45$

14. Dangeard (L.) et Hommeril (P.), 1964. - Le grès calcaire normannien de Bernières-sur-Mer (Calvados) et les dépôts associés. Datation au carbone 14. - C.R. somm. Soc. géol. France, 16 mars (3), p. 126.

15. Gull laume (L.), 1948. - Les formations quaternaires de la plage de débarquement britannique de Saint-Cóme de Fresné, Asnelles-Belle-Plage (Calvados). - Rep. 18 th Sess, int. Congr. Great-Britain, part 13, p. 105-112 
16. Hommeril (P.), 1958. - Erosion et sédimentation à Saint-Aubin-sur-Mer. - Bull. Inf. Com. Océanogr. Et. côtes. 10 (9) et (10). p. 559-616 ct 691-740.

17. - 1961. - Méthodes d'étude de la granulométrie des formations à galets. Application préliminaire à quelques cordons du littoral nord du Massif de Barfleur (Manche). - Bull. Soc. linn. Normandie, 10 sér. 2, p. 242257.

18. KIng (W. B. R.), 1954. - The geological history of the english Channel, - Quart. J. géol. Soc., Londres, 110, p. $77-102$

19. Rıult (M.), 1960, - Sur un galet de poudingue à nummulites remaniées. - Bull. Soc. linn. Normandie, I. p. I $46-148$. 